\title{
Apicultura, entorno y modernidad en localidades de Yucatán, México
}

\author{
Enrique Rodríguez Balam \\ Miguel Pinkus Rendón * \\ Centro Peninsular en Humanidades y en Ciencias Sociales \\ Universidad Nacional Autónoma de México \\ Calle 43, s/n, Col Industrial, C.P. 97150, Mérida, Yucatán, México \\ * Autor para correspondencia \\ mapinkus@humanidades.unam.mx
}

Submetido em 08/09/2014

Aceito para submissão em 27/05/2015

\section{Resumo}

Apicultura, ambiente e modernidade em localidades de Yucatán, México. O presente trabalho faz uma reflexão acerca do conhecimento local dos apicultores a respeito da abelha europeia Apis mellifera scutellata, sua biologia, comportamento, estrutura social, comunicação e a relação que estes organismos têm com o ambiente, seus inimigos naturais e o impacto que tem o uso do solo nesta atividade econômica. Este conhecimento empírico converge de maneira muito acertada com o conhecimento científico que se tem de este grupo de organismos.

Palavras-chave: Apis mellifera; Conhecimento local; Relação homem-natureza

\section{Abstract}

Beekeeping, environment and modernity in localities in Yucatan, Mexico. In this paper, we reflect on the local knowledge about the European honey bee Apis mellifera scutellata, namely its biology, behavior, social structure, communication, and the relationships that these organisms maintain with the environment and their natural enemies. We also discuss the impacts that land use has on this economic activity. The empirical knowledge of beekeepers converges quite well with the scientific knowledge concerning this group of organisms.

Key words: Apis mellifera; Local knowledge; Human-nature relationship

\section{Introducción}

\section{De la tradición a la modernidad}

La relación de los pobladores de Yucatán con el entorno y en particular el trabajo vinculado al espacio que denominan "monte", ha sido desde antiguo un referente para entender formas culturales de adaptarse a un medio cada vez más cambiante (TOLEDO et al., 2008). Del mismo modo, las actividades productivas que se derivan del vínculo con la naturaleza nos hablan también de formas de organización social, identidad, economía, pero sobre todo, de una constante actualización de un acervo de conocimientos sobre el ambiente y la manera de aprovechar de él los recursos necesarios para subsistir; saberes y tecnologías que se 
han transmitido a través del tiempo, para enmarcarse dentro de aquello que se ha denominado "tradición".

Como sucede hoy en día, la apicultura se encuentra inmersa en una transición que va de lo tradicional a conocimientos y usos de herramientas tecnológicas, que se enmarcan dentro de la modernidad. En el caso de los entrevistados en nuestra indagatoria, los resultados encontrados arrojaron información que dejaba entre ver que ambas categorías - tradicional vs modernidad - se encontraban contrapuestas, particularmente en el orden discursivo; no así en el de la práctica productiva. Como ejemplo de lo anterior, queremos apuntar que para el caso específico de éste artículo, llamamos "tradicional" a las técnicas, métodos y conocimiento adquirido de generación en generación por los apicultores. "Moderno" y "modernidad" en el mismo caso, se refieren al uso, no sólo de nuevas tecnologías, sino también al conjunto de discursos construidos desde el ambientalismo. Debido a la riqueza analítica que ofrece la referencia al "ambientalismo", abordaremos el tema en apartados posteriores.

Como suele ocurrir, tanto el uso de conocimientos del entorno desde visiones tradicionalistas y modernas, no se construyen sobre procesos unilineales. De hecho, es frecuente que convivan ambos tipos de conocimiento. Con lo anterior, queremos señalar que la profundidad cultural de una actividad productiva como la apicultura se expresa en las formas de mantener un conjunto de conocimientos sobre el entorno, derivados del aprendizaje vía oral a través de sus padres y abuelos, pero también en la adaptación de nuevas formas provenientes de esquemas no tradicionales. Sobre ésta idea referimos la siguiente cita:

"Aunque tradición sigue implicando profundidad temporal, así como continuidad, los especialistas actuales están llegando a verla, no como una cualidad innata, imbuida de continuidad y éstasis, sino más bien como un imperativo de la vida social, una forma en la que el presente interpreta y caracteriza el pasado, pensando en el futuro". (BARFIELD, 2000).

Desde nuestra perspectiva y de acuerdo con la información obtenida, la tradición y lo tradicional hacen referencia también a la puesta en práctica dentro de la vida diaria - incluidas las actividades productivas - de un conocimiento histórico sobre el entorno, pero también de nuevas prácticas y tecnologías modernas.

Actividades como la cacería de los venados yис o temazate (Mazama americana) (Erxleben, 1777) y keh o cola blanca (Odocoileus virginianus) Zimmermann, 1780, principalmente, así como la captura de aves para su comercio y la apicultura son muestra de ello (MANDUJANO; RICO-GRAY, 1991; SANTOSFITA et al., 2012). De este tipo de labores vinculadas con el medio, llama la atención que sobre todo para la así denominada "área maya yucateca", no se les haya prestado atención desde el punto de vista cultural. En muchos sentidos, se ha dado más peso a la importancia cultural e histórica del trabajo en la milpa y los rituales agrícolas que generalmente les acompañan, en tanto que se entienden como parte del "contexto maya". Razón por la cual, pareciera que tales prácticas han sido elevadas a rango de valor "cultural" para casi toda el área mesoamericana. Trabajos recientes son ejemplo de lo anterior, debido a que algunos se han producido exclusivamente para hablar sobre la milpa como espacio indispensable para comprender el contexto cultural de los mayas peninsulares (TERÁN; RASMUSSEN, 1994).

Quizá ello justifique el hecho de que la información con la que contamos para vincular disciplinas como la antropología, la etnoecología o etnobiología, se apoya más desde los aportes proporcionados por biólogos que por científicos sociales (TOLEDO et al., 2013). Salvo escasas excepciones, no contamos en fechas recientes con trabajos etnográficos que se hayan dedicado a abordar temas como la cacería o la apicultura, pues en la mayoría de los casos, apenas se han logrado conjuntar datos dentro de algunos apartados y no como objetivo central de una investigación total.

A pesar de lo enunciado en párrafos anteriores, el trabajo de campo muestra que en buena parte de los municipios y comisarías del estado de Yucatán, todavía se mantienen formas tradicionales de llevar a cabo tales prácticas productivas, mediante el empleo de nuevas técnicas. Para el caso particular de este artículo, nuestra intención es enfocarnos en los mecanismos de transmisión de conocimientos y tecnologías de padres a hijos, pero sobre todo, la manera en la que una actividad como la apicultura mantiene elementos y técnicas 
tradicionales, así como aquellas que requieren hoy en día los modernos procesos de producción de miel entre gran parte de los apicultores peninsulares.

En el mismo sentido, se trata de mostrar la manera en la que las constantes adaptaciones derivadas de aprendizajes nuevos, responden a demandas de orden global y que terminan por modificar percepciones y discursos locales. Discursos emanados de ideologías ecologistas, así como enunciados que buscan encajar dentro de esquemas conservacionistas, también han sido adaptados dentro de los usos de vocablos de los apicultores actuales, en convivencia con visiones del mundo desde sus referentes tradicionales, más apegados a aquello que se denomina "tradición".

Si bien la apicultura o uso productivo de la Apis mellifera Linnaeus, 1758 (Hymenoptera, Apidae) fue introducida entre 1760 y 1770 a la Nueva España debido a la necesidad para contar con un mayor suministro de cera para las cuestiones religiosas (LABOUGLE; ZOZAYA, 1986). Este tipo de actividad había sido realizada por los grupos mayas desde el período prehispánico, aunque en realidad se utilizaban abejas sin aguijón Trigona spp. y Melipona beecheii Bennet, 1831, si bien existe un gran número de meliponinos en Yucatán (QUEZADA, 2005), ésta última especie era la que más se menciona en los textos históricos. Se ha relatado que estas abejas eran cuidadas por los mayas para la producción de miel para endulzar tanto alimentos como bebidas, que eran usadas tanto en el cotidiano como para uso ritual (balché). Ésta bebida, a base de miel fermentada y corteza del árbol de balché, fue utilizada en todas las ceremonias mayas, ya que era un líquido asociado a la purificación, además de producir estados alternos de conciencia donde se develaban misterios que únicamente eran mostrados en este estado (BERNARD; LOZANO, 2003). Incluso se considera a uno de los dioses, $\boldsymbol{A} \boldsymbol{h}$ mucen cab (Divina Abeja Roja) como protector de las abejas, deidad que puede observarse en el códice Tro Cortesiano (RAMOS-ELORDUY et al., 2009). Rivera y Cappas e Sousa (1998) mencionan que para los mayas prehispánicos las abejas eran hacedoras de la fertilidad y por ello cuando morían, debían ser enterradas para que en el mundo de la muerte construyan vida a partir de cosas muertas. Por lo anterior, es que suponemos que la A. mellifera fue bien aceptada por los grupos que cosechaban miel, sobre todo por la cantidad de producto que puede ser extraído de las colmenas, principal diferencia con las abejas nativas.

Es así que la idea que se mantiene a lo largo del texto es que con independencia del tipo de técnicas, conocimientos o herramientas empleadas, la apicultura es una actividad económica que mantiene elementos históricos y culturales que, contrario a lo que se suele creer, se vinculan con el pasado; aspecto relevante que nos permite entender por qué se mantienen ciertos rasgos culturales, en la actualidad. Algunos, incluso, con mayor peso respecto de otras actividades económicas tenidas por "tradicionales", como sería el caso de la agricultura, particularmente el cultivo del maíz. Es decir, que la agricultura de la milpa (Roza-tumba y quema) no ha sido la única actividad económica que ha perdurado a lo largo de los siglos en Yucatán.

\section{Material y Métodos}

La recopilación de la información se realizó de enero de 2012 a junio de 2013 en los municipios de la costa norte de Yucatán (Sinanché, Yobaín y Dzidzantún), así como del sur (Tekax, Oxkutzcab y Tzucacab) ${ }^{1}$ y oriente del estado (Tizimín, Valladolid) (Figura 1). Para lo cual se llevaron a cabo 20 entrevistas semiestructuradas a apicultores hombres de entre 18 a 70 años de edad (aproximadamente dos por localidad), esto debido a que en las localidades de estudio, la gente atribuía las actividades vinculadas a la apicultura como práctica exclusiva de los hombres. Por ello, se solicitó un permiso formal a los mismos para participar en esta investigación con la finalidad de que la información obtenida a partir de sus narraciones, estuviese en apego a los estándares éticos internacionales de la etnobiología. Cabe señalar que la Península de Yucatán es la mayor productora de miel

\footnotetext{
El presente estudio estuvo enmarcado dentro del proyecto "Domesticar la biodiversidad. Concepción y empleo de los recursos naturales en la costa central y el sur de Yucatán" financiado por el FOMIX CONACYT-Yucatán 2008 bajo la clave 108904.
} 
FIGURA 1: Sitio de estudio. En el mapa se muestran las localidades y municipios dentro del Estado de Yucatán, México donde se aplicaron las entrevistas.

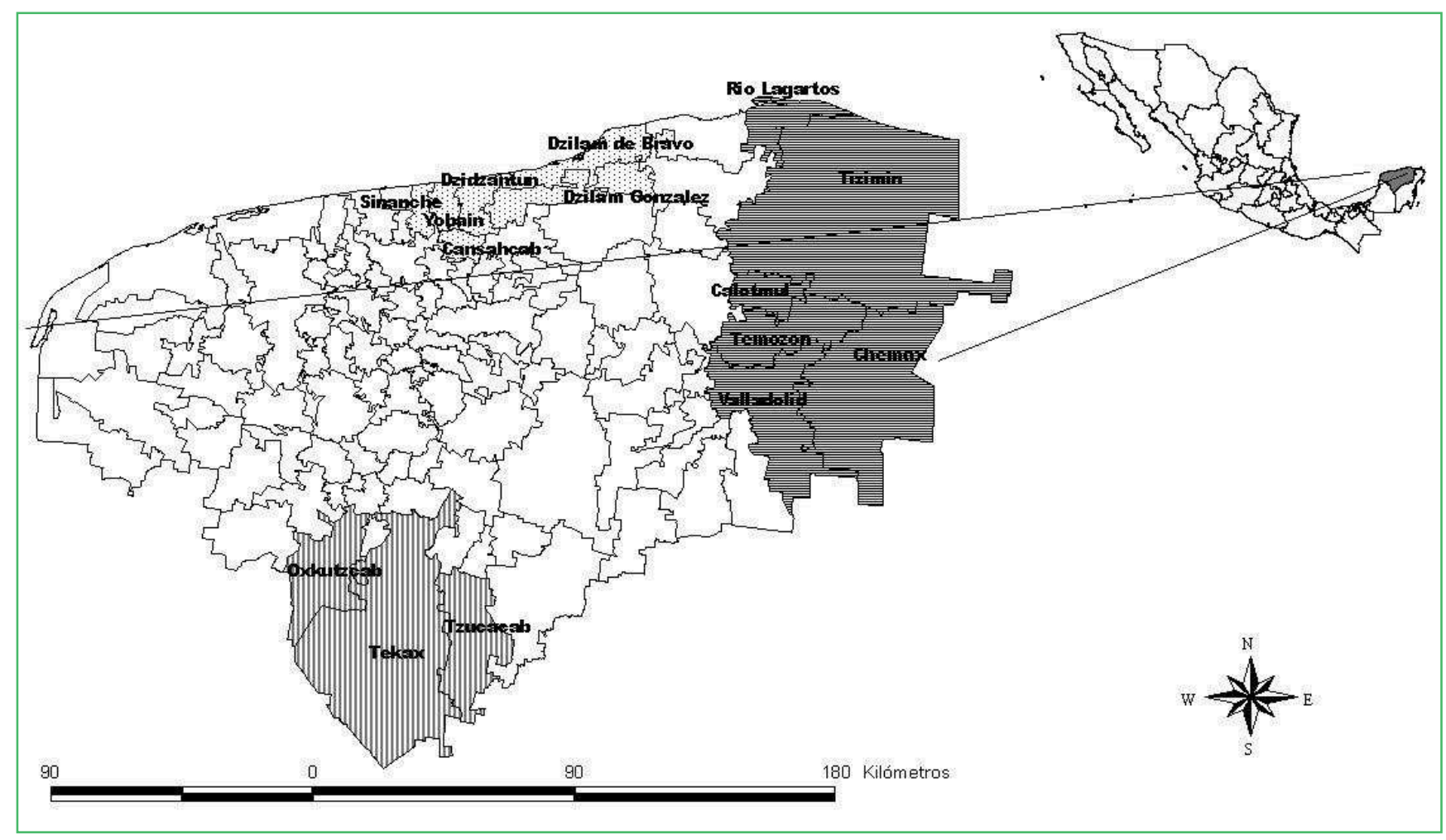

en el país, así también, el promedio de colmenas por apicultor es de 20; no obstante, en algunas localidades del oriente (Tizimín y Valladolid) pueden llegar hasta 900 colmenas (PINKUS, 2013a). En las entrevistas cualitativas se abordaron las temáticas referentes al conocimiento local que tienen los apicultores respecto a la Apis mellifera y otras especies nativas de abejas (biología, ecología, comportamiento y padecimientos). Para la identificación de las abejas se recurrió claves taxonómicas del grupo (AYALA, 1999). Asimismo, abordamos la inclusión que estos organismos tienen y han tenido en la cosmovisión de los pobladores, así como el uso que se le ha conferido a las especies o sus productos empleados en rituales. Si bien en las descripciones etnográficas se ha asumido que este término se relaciona con cosmogonía y cosmología, en lo concerniente a nuestro trabajo se refiere, stricto sensu, a la visión del mundo, referida particularmente al ámbito de la vida cotidiana y la transmisión de conocimientos.

\section{Resultados y Discusión}

\section{Identidad y apicultura}

Si partimos de las teorías ecológicas que indican que el entorno se conforma de manera importante por la manera como una sociedad se relaciona con él, quizá podamos colegir que la apicultura ha representado una de las actividades más antiguas que también han demostrado ser autosustentable pues - ya lo veremos más adelante - requieren de un equilibrio ecológico para poder subsistir (MELIC, 2002). Es decir, existe un ciclo en el que se aprovecha la miel que se produce para el consumo directo de las familias y los remanentes se venden. Para ello es imprescindible que no se promuevan transformaciones en el entorno por el cambio del uso del suelo, para asegurar la disponibilidad de los recursos melíferos para las abejas. De ahí que podamos sostener la hipótesis, que además de los elementos culturales e identitarios que han servido de referente para generar "cultura" por parte de los sujetos, la actividad 
económica (apicultura) es otro factor de identidad que preserva de manera integral el entorno natural, han sido los mecanismos que han podido garantizar, hasta el momento, que ésta se mantenga como actividad productiva y práctica cultural, ya que en un $80 \%$ de las localidades yucatecas cuenta con, por lo menos, veinte colmenas de abejas por familia. Incluso en lugares con alta producción como el sur y oriente de Yucatán casi la totalidad de las familias poseen abejas. Hoy en día, ciertas formas de trabajar han tenido continuidad, a pesar que en la gran mayoría de los casos, tanto los jobones como las técnicas empleadas para trabajar con las abejas nativas, han cedido terreno a las nuevas tecnologías empleadas hoy en día por los apicultores del estado, entre las que se incluyen las nuevas cajas para abejas, el control biológico para luchar contra la varroasis o el uso del traje completo de apicultura.

Tal como lo sugerimos en párrafos anteriores, la extracción de miel es una actividad que ha estado presente entre los pueblos mayas desde épocas prehispánicas. De ello nos da luz la información que se desprende de Códices - El Códice Madrid quizá sea el más emblemático, grabados, glifos, pero también lo que textos mayas tales como el Chilam Balam de Chumayel y documentos coloniales, nos constatan respecto de la antigüedad de dicha práctica.

Voces y conocimientos se ponen en juego a la hora de interpretar el mundo y la naturaleza, valores culturales que proveen de formas de interpretar también la interacción social de los sujetos dentro de sus propios contextos sociales. Visiones de mundo que pueden incluso designar el "género" de insectos como el de las abejas a quienes se les considera mujeres, diferenciadas también por edades: a algunas se les considera señoras, madres, y otras, niñas. Es decir, en algunas ocasiones se antropomorfiza a los organismos designándoles propiedades características de los humanos (MORALES, 2010), que en este caso, señalarían las etapas del comportamiento de las abejas adultas.

A caso sirva como ejemplo de lo anterior el hecho de que un apicultor de Dzidzantún, Yucatán explique su manera de llamar a sus abejas de la siguiente forma: "Yo les hablo con cariño, desde temprano, porque son inteligentes y además sienten lo que uno les dice, sienten el tono, la vibración, les digo: - Hola niñas, buenos días, ¿Cómo están? ¿Están bien, chiquitas? - Así también hay unas que son como las mamás de todas, son las jefas".

\section{Reconocimiento del entorno, saberes locales}

Como se ha referido en otros trabajos, la relación del hombre con el monte es fundamental. Aquello que una descripción a distancia podría referirnos para buena parte del territorio yucateco, se basa en la idea que éste se encuentra rodeado principalmente de monte bajo, o selva baja subcaducifolia, plagado de plantas arbustivas y fondeado por un suelo denominado litosol o rendzina, de piedra caliza (laja). Sin embargo, sabemos que existen variedades tanto en vegetación como en tipos de suelo dentro del mismo territorio estatal y que, dependiendo de la región de la que hablemos, será el tipo de entorno (flora $\mathrm{y}$ fauna) al que hagamos referencia (BAUTISTA et al., 2005). Más allá de aspectos con tal especificidad, para la gente que trabaja en el campo, adentrarse en el monte requiere desarrollar un tipo de sensibilidad y adquisición de conocimientos que son fundamentales para sobrevivir y poder extraer de la naturaleza lo necesario para la vida diaria (RODRÍGUEZ-BALAM, 2010a).

Recorrer el monte, o ir a "montear", saber reconocer qué posibilidades proporciona para la siembra, saber de temporadas, de vientos, son saberes imprescindibles para el desmonte, ${ }^{2}$ el cultivo o cualquiera de las actividades que se trate. Son habilidades que todo aquel que quiera obtener recursos de la naturaleza debe tener. Reconocer los tipos de plantas, árboles, el tipo de suelo, así como el uso que se les puede dar, son tan sólo algunos elementos básicos a la hora de comenzar una actividad dentro de dicho espacio natural. Precisa saber de todo, incluso de historias, leyendas y creencias.

Por ello es que los hombres deben saber reconocer ciertas plantas, porque algunas de ellas sirven para curarse de hechizos y encantamientos que su mujer pueda imponerles. Cuando hay mucho tábano, por ejemplo, será señal que se encuentra rodeado por una buena cantidad del árbol llamado chucúm (Havardia

\footnotetext{
Cortar maleza.
} 
albicans) (Kunth) Britton y Rose, en florecimiento. Es decir, vinculan una relación entre el comienzo de la floración de esta planta y el brote de los dípteros adultos. Cerca de esta vegetación también se encuentra la mata de surím o subín (Acacia cornigera) (L.) Willd., la cual está rodeada de hormigas rojas, conocidas como "hormigas de subín" (Solenopsis gemminata, Fabricius, 1804), que se caracterizan porque "pican fuerte" (PINKUS, 2013b). Esas hormigas sirven precisamente para despertar al marido del hechizo que le ha hecho su mujer. De otro modo, el esposo no tendrá la capacidad de percibir la infidelidad de la mujer, incluso cuando ésta lo haga en su presencia. Por ello es que también, aunque la referencia sea en tono de broma, tampoco debe fiarse de un hombre que llegue a tocar a la puerta, pues podría tratarse del "sancho"3 [Sic] transformado en Uay chivo. ${ }^{4}$ Sobre ello algunos informantes nos dicen:

"Hay plantas que las mujeres usan para hacer hechizos a sus maridos. Hay mujeres que son infieles, dicen, ta' curado, el marido puede ver que la esposa esté con otro y él ni en cuenta, dicen que con estas hormigas hacen que despierte el marido, ya sea que lo coma o que lo tome con una bebida, para que despierte. Es la hormiga del subín. Es su hábitat natural. La mata tiene cuernitos con huecos y ahí se meten, la defienden, defienden a la planta, las hormigas defienden a la planta de todo, si te pegas, te pican y duele y tiene espina."

Más allá de este tipo de creencias, la diversidad de plantas y formas de emplearlas, son más complejas de lo que se puede observar. Los árboles que se pueden reconocer con mayor "facilidad", según nos lo cuentan los entrevistados, son el dzidzilché (Gymnopodium floribundum) Rolfe, chucúm (H. albicans) y tzalam (Lysiloma latisiliquum) (L.) Benth., apreciados por los apicultores ya que son árboles que pueden mantener la humedad por más tiempo y así permanecer en buen estado a pesar de que las precipitaciones pluviales no sean frecuentes. Dicha condición en la flora será siempre bien vista por quienes cuentan con apiarios, ya que estos

\footnotetext{
3 Nombre popular para designar al amante de la esposa.

4 Según referencias etnográficas, es la capacidad que tiene un especialista ritual para transformarse en animal o ser del monte. En algunas localidades, se ha difundido la creencia que incluso una persona vinculada con actos de brujería es capaz de mutar de hombre a animal.
}

tipos de árboles ayudan a mantener la temperatura y humedad necesaria para las abejas.

El bejuco, cuyo nombre en maya es solenak (Bonamia brevipedicellata) Myint y D.B.Ward, da flores blancas y es apreciado por ello para la apicultura pues se considera melífera, al igual que todo el entorno, pues con independencia de su utilidad para la polinización, funcionan como fuentes naturales de refugio para las abejas. Dentro de dichos contextos naturales (hábitats) también se entiende la existencia de determinado tipo de animales que buscan también refugio en los espacios más equilibrados ecológicamente. Así, es posible ver merodear o aparecer entre los caminos del monte culebras, pizotes, venados, pavos de monte, codornices, gallinas. Sin embargo, y como es habitual, también existen depredadores de las abejas. Como lo comenta el entrevistado, el sanjol (en maya) Eira barbara Linneo, 1758, es un animal como "especie de oso hormiguero" que come abejas y anda en busca de larvas.

También existen armadillos y el "póleo" (Procyon lotor) Linneo, 1758, que es según los agricultores “como un perrito que tiene como unos lentes negros. Es parecido al mapache, ese viene de la familia de los mapaches. El póleo su especialidad son los elotes, anda cerca de las milpas y las sandías".

Del mismo modo, entre los límites de Dzidzantún con Cansahcab, se observan nidos de pájaros, las "yuyas" como le denominan algunas personas. Es común que este tipo de animales se observen cerca de áreas llenas de árboles de gran tamaño como los jabines, o el tzalam, incluso algunos donde se encuentre el árbol denominado álamo, puede ser indicador dentro del paisaje que existen depósitos de agua naturales o cenotes.

Además de dichos lugares con agua, las lluvias también dejan salir muchos insectos que se aprovechan de la humedad tales como el gusano llamado "cogollero" o "Nok'ol" por su nombre en maya (Spodoptera frugiperda) Walker, que sale cuando deja de llover. Según nos explica uno de los agricultores, el entorno natural está ordenado de acuerdo a sus funciones, como si de una sintaxis narrativa se tratase, ya que así como hay árboles que nos dicen de la cercanía de cenotes, o de cierto tipo de animales. También existen referentes naturales que se 
relacionan con seres del monte, como la Xtabay -según las leyendas mayas, se trata de una mujer que se aparece cerca de una Ceiba sp. que tiene el poder de perder a los hombres ebrios-, que está vinculada a árboles sagrados (considerado así desde épocas prehispánicas) como las ceibas. Sobre ello respondía un agricultor al preguntarle qué hacía "Aquí estoy. Sembrando mata de Ceiba acá, para que se aparezca la Xtabay".

Si fuese nuestra intención establecer un vínculo entre el binomio naturaleza-cultura, como se deriva de ciertos estudios antropológicos, podríamos decir - desde la perspectiva de los apicultores locales - que los nombres de plantas y animales en lengua maya han desaparecido, no sólo por los procesos de transformación cultural habituales (empleo asalariado, abandono del campo y migración entre otros), sino también porque las actividades productivas, tales como la ganadería, han destruido el hábitat. Si con el trabajo en el monte los saberes del entorno se han mantenido, la creciente cantidad de ranchos, con actividades "ajenas" a las "tradicionales", han contribuido a dejar en el olvido prácticas, nombres y clasificadores en lengua materna. En ese sentido, además de los taxones locales, conocimientos respecto al entorno y la construcción de apiarios, han tenido que transformarse con el paso del tiempo y el ingreso de nuevas tecnologías (ECHAZARRETA, 2010).

\section{Conocimiento local y manejo de las abejas}

Según lo describen los apicultores yucatecos, el primer paso para poder construir un apiario que cumpla con los requerimientos mínimos, consiste en ubicar un espacio que cuente con cenotes o fuentes de agua cercanas, así como diversidad de flora y fauna, se procede a levantar el apiario. Dentro del esquema actual, resulta conveniente poner en juego todo el conocimiento con el que se cuente. La idea de contar con un ambiente diverso radica en que se pueda mantener de manera constante los ciclos de floración durante todo el año, o períodos prolongados, siempre que ello sea posible. Sobre ello nos explica un apicultor (38 años):
"El proceso inicia de la siguiente manera: Primero, lo que te dije, buscar un lugar como este, que tenga árboles, que sean capaces de darnos el suficiente néctar para poder producir cantidades de miel, variedad, ¿por qué?, pues porque no todos florecen al mismo tiempo y eso garantiza que seguido haya para sacar miel. Ahorita florece el chucúm; el jabín (Piscidia piscipula) (L.) Sarg, en abril, dzidilché; enredaderas en noviembre; el tajonal (Viguiera dentata) (Cav.) Spreng., en enero; entonces hay un ciclo ininterrumpido. Cuando cae mucha lluvia, es bueno, porque la flor del chucúm y el catzin, les queda humedad para un buen tiempo.

Después de este proceso, es necesario comenzar a "ubicar" el apiario, tratando en lo posible de tenerlo en lo que la gente conoce como "altillos", pequeños montículos sobre el suelo que permiten colocar el apiario a una altura que los pueda proteger de animales depredadores, pero también de inundaciones por lluvias o tormentas.

Después empiezas a ubicar tu apiario. Si te das cuenta es un altillo, porqué... llueve mucho, se inunda, como está en altillo no les llega el agua. Cómo está ubicado, las piqueras están viendo de este lado, cuándo sale el sol, los primeros rallos les dan a las piqueras y salen al campo. Lo otro es que estén del lado contrario de los vientos. Cuando pega un norte, no les entra el lado frío, ahora si viene caliente tampoco les entra directo, entonces, eso sí nos lo enseñaron los antiguos, ya empiezas a comprar tus cajas, posicionar tus colmenas. Puedes comprarlas, una dos tres colmenas, para que vayas haciendo divisiones..."

Tomando en cuenta los conocimientos requeridos sobre el entorno para dedicarse a la apicultura, es de tomar en consideración el hecho que sean los mismos apicultores quienes señalen que, actividades tradicionalmente relacionadas con el monte, asumidas desde ámbitos antropológicos como garantes de su continuidad cultural e identitaria, sean las que exterminen el entorno que les rodea, pues como los mismos apicultores nos dicen, es preciso localizar, desde el inicio, cuántas milpas tienes alrededor, ya que "Cuando alguien quiere hacer milpa, acaba con la variedad que hay". Cabe señalar que con la agricultura de roza, tumba y quema, se cortan muchas plantas melíferas, dejando solo aquellas que posteriormente puedan ser utilizadas. Bajo el mismo argumento, los apicultores apuntan que su actividad tiene el mismo peso histórico que la agricultura dedicada al maíz. 
Al igual que la siembra de maíz afecta a las poblaciones de abejas, otra actividad que es mucho más agresiva tanto para el manejo de abejas nativas como para la apicultura es la ganadería, no solo porque se devastan grandes extensiones de terreno para la introducción de ganado, sino porque tratan de erradicar plantas como el tajonal que compite por espacio con los pastizales inducidos (VILLANUEVA et al., 2005).

Una vez que se ha pasado este proceso, comienza el trabajo para lograr que las abejas hagan las labores que les corresponden. Así, la colmena debe contar con panales, algunas crías, así como con abejas reina y miel. En un inicio, las abejas salen a inspeccionar su área sin alejarse mucho del punto donde se encuentra el apiario (Figura 2). Ya una vez orientadas, van aumentando el rango y tiempo de recorrido. Así, durante los primeros dos días, vuelan $3 \mathrm{~km}$ de distancia y regresan. Esta práctica la llevan a cabo durante dos o tres semanas aproximadamente, hasta que son capaces de poder viajar grandes distancias para conseguir un mejor trabajo de polinización y producción de miel.

En el interior de las "cajas", existen tipos de jerarquías a través de las cuales se organizan las abejas y el trabajo que realizan. Abejas reinas, zánganos y pecoreadoras, son algunas de las formas de nombrar y designar, tanto jerarquías y ordenamiento "social" en el interior, así como las actividades que realizan. Sobre ello nos dicen los apicultores:
"El primero, la primera caja, viene lo que es la cámara de cría, es donde está concentrada la reina, las obreras nodrizas, la cría, lo fuerte, zánganos y todo. Lo demás son alzas, nos sirven para cosechas, cuando vayamos a cosechar no nos sirve la cámara de crías. Aquí está la reina y eso no se toca, eso hace que la colmena se pueble, mientras más población más néctar. La abeja tarda en brotar treinta días, los primero días, son nodrizas, niñas, luego son guardianas, las que están el piquera a ver quien [sic] entra, luego las tareas de limpieza, las más adultas, las pecoreadoras son las que se van al campo, van al campo y traen néctar, flujo de

FIGURA 2: Apiario sobre rieles para evitar el ataque de enemigos naturales. En la figura aparece la forma en que los apicultores colocan sus apiarios sobre rieles de tren para evitar el ataque de hormigas y otros enemigos naturales.

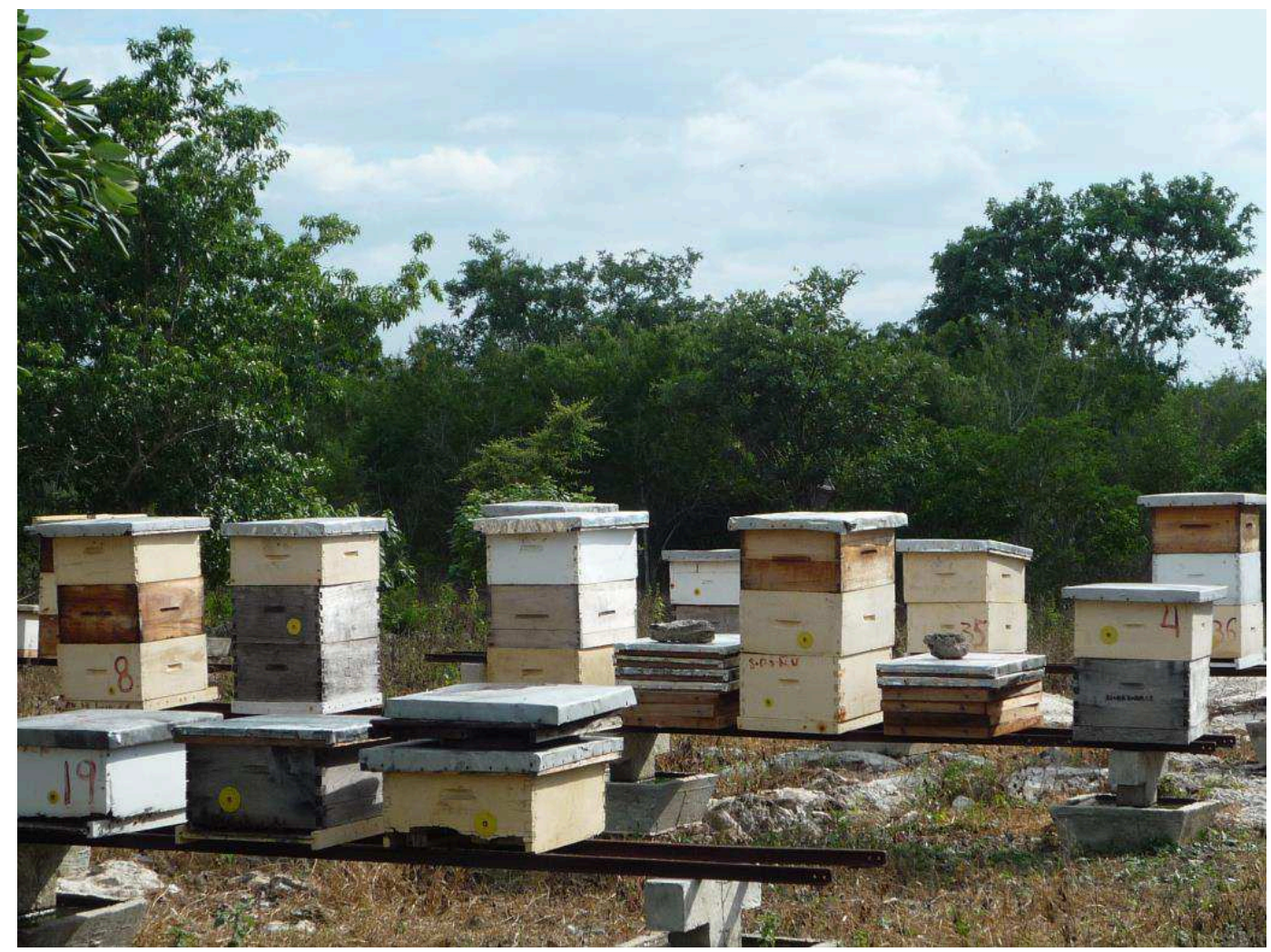


miel, es la última etapa porque de allá, mueren. En la caja de arriba están las pecoreadoras, porque traen y suben. En la primera caja su trabajo es mantener a la cría, le dan calor, tiene que mantener su temperatura. El zángano sólo fecunda a la reina porque no va al campo. Pero cuando hay escasez de néctar va para afuera, lo sacan y los matan, se mueren de inanición. Es que la abeja prevé. Cuando va a empezar el flujo de néctar la abeja pone a discreción cuando se está acabando baja su postura. Los zánganos sólo comen miel, como son grande acaban con reserva y se quedan sin nada, primero, se van para afuera y aunque quieran entrar, no los dejan y ahí se mueren, no saben pecorear. Sus labores están bien establecidas. Cómo conoces las funciones, en parte porque me lo ha contado mi papá, toda su tradición de apicultor, toda su vida".

Este conocimiento empírico concuerda plenamente con las etapas conductuales que se desarrollan a partir de la operculación de los huevecillos, en los que incluso se ubican la limpieza de las colmenas de parásitos tales como el ácaro Varroa destructor Anderson y Trueman, 2000 (VANDAME et al., 2000).

Además de tan variados y complejos procesos de orden y estructura en la organización social de las abejas, tanto los depredadores animales como los fenómenos meteorológicos pueden provocar que las cajas queden "huérfanas". Al perder a la abeja reina, las abejas pueden llegar a estresarse y con ello, urgen la necesidad de poblarse, con el riesgo de volverse todas zánganas pues la abeja comienza a ovar, pero los huevos no brotan. Con acotada precisión los apicultores nos narran:

"La caja queda huérfana, no tiene mamá, ¿qué hacen? Se estresan las abejas y al estresarse tienen que ver cómo poblarse entonces se vuelven zanganeras porque la abeja empieza a ovar, pero esos huevos no brotan. Porque no son fértiles, entonces la colmena empieza a morir la abeja adulta. La reina tiene su función de poner larvas, las abejas se encargan de todo, es más, a la reina la alimentan con lo mejor, miel polen.

Las abejas trabajan durante todo el día, incluso algunas, es posible que tras haber recorrido un largo trayecto, no alcancen a regresar, por lo que se quedan pegadas a los árboles para emprender el viaje de retorno al día siguiente. La capacidad que tienen para reconocer los mapas que se crean para el retorno, son posibles gracias a que cuentan con "antenitas, son como su brújula, ya tienen ubicado la distancia, cuando salgan ya saben por dónde se van, son bien ubicadas. Nosotros nos podemos ir pero ya sabes cómo regresar, aunque estés tomado regresas y si te agarró la noche te quedas".

Es así que los apicultores explican el comportamiento de comunicación de las abejas, en la que se da una danza conocida como "el ocho", en la cual la abeja pecoreadora transmite la información de la disponibilidad de alimento, distancia y orientación en la que se localizan las flores (ACEÑA, 1990).

La anatomía de la abeja la ha provisto de elementos que tienen diversas funciones para su preservación y trabajo tales como la capacidad de ubicación espacial y cálculo de distancias largas. Asimismo, son capaces de emitir sonidos desde las colmenas, que indican a aquellas que se han ido a polinizar, saber reconocer dónde se encuentra el resto de las abejas para así poder dirigir su trayecto y regresar al apiario. Incluso en épocas de calor, o bien cuando se preparan para enjambrar, realizan danzas de extraordinaria coordinación. Los apicultores comentan:

"En tiempos de calor empiezan a ventilarse, tienen sus danzas cuando van a enjambrar, jalan la piquera, se mueven, vuelan sobre las cajas, sólo se mueven, es como danza sin volar, hacen figuras van para arriba, vienen, es señal que se preparan para enjambrar, es cuando está fuerte la colmena, están sobre poblados y se requiere que se vaya una parte, entonces hacen reinas y cuando brote la primera o la segunda, se van, salen enjambres cuando ven las abejas... entonces así ya se puede formar una colmena, es otra... una colmena sólo tiene una reina, no puede tener dos, es como la casa sólo una mamá debe haber, no dos... en casas sí hay... suele suceder... qué pasa cuando hay dos reinas, cuando chocan, ya a pelear, puede ser que muera una puede que mueran las dos".

Quizás resulte conveniente mencionar que la apicultura, como actividad de arraigo histórico entre las poblaciones de Yucatán, ha pasado por diversas transformaciones hasta su revaloración actual como actividad productiva, pero también en cuanto a las modificaciones que han tenido las técnicas para desarrollarla (LABOUGLE; ZOZAYA, 1986). De ahí que algunos señalen que la apicultura comenzó desde épocas pasadas, de cuando no existía la fertilización que tanto daño ocasiona al ecosistema, de cuando se trabajaba sin guantes, sin velo y las colmenas estaban en los solares de las casas. En esos tiempos, según 
recuerdan, existía gran diversidad de abejas, algunas que ya ni por nombre se conocen en la actualidad. Apuntan que fue a partir del año 1985, cuando se modificó todo gracias a la "africanización" de las abejas. Sobre ello también asientan:

"La apicultura empezó desde hace años, antes de que venga la fertilización, sin guantes sin velo trabajábamos en el apiario. Desde el 85 cambió todo con la africanización, antes entrabas sin velo, ahora cambió todo, a veces hasta en las casas se tenían las colmenas. Recuerdo que mi papá tuvo cuarenta colmenas en el patio de la casa, pero sin que sean tan agresivas, pero desde que llegó la africanización cambió y pusieron reglamentos de que debe estar fuera de la población para no perjudicar.

Apis melifera. Así se llama, su nombre en maya no las conozco así, porque no me lo han dicho... porque los antiguos que trabajaban la apicultura casi no hay. Xunancab (Melipona beecheii), siempre pequeña sin aguijón. Esa no se trabaja aquí. Posiblemente haya esa abeja, en el norte donde nadie llega, pero aquí no hay... xuxil, la viejita, lo que se ve a simple vista, tiene sus temporadas también el xux. El nikchac (Polistes sp.), es una avispa que hace sus panales en árboles para lluvia, panales inmensos, tremendas avispas, ese sí, nikchac es su nombre, si le pegas sin querer, te dan una curtida, con tres picadas que te den una calentura que te da porque es fuerte, es muy agresiva".

En la actualidad, debido a la modificación que ha sufrido la apicultura así como su revalorización en los mercados internacionales; su producción, costos y comercialización, han tenido un impacto en la cantidad de productores que se dedican a dicha actividad. Hoy en día, tan sólo el municipio de Dzidzantún cuenta con cerca de 40 apicultores, de los cuales, el $80 \%$ se dedica al trabajo con la abeja africanizada y sólo 20 a la apicultura orgánica. No obstante, el número de colmenas por persona varía, ya que dependiendo la región será el número de colmenas que tengan en su monte, alcanzando en algunos casos (Tizimín) hasta 900 colmenas. En el caso de la mayoría de los apicultores, se trata de gente adulta que más allá del ingreso que puedan obtener, mantienen dicha actividad por mantener la "costumbre", o bien, porque sus padres les enseñaron a "trabajar la abeja". Por ello, hay incluso algunos que optan por contratar a otras personas para que cuiden y mantengan sus apiarios.
En términos de cantidad, la miel se mide en kilos. La abeja reina es capaz por sí sola de poner cerca de 1.500 huevos al día. En ese sentido, si la inversión del equipo no rebasa los 500 pesos, y dado que una colmena produce entre 30 y $40 \mathrm{~kg}$ de miel, que tienen un costo de 35 pesos, es posible recuperar un poco más del 50\% de la inversión inicial. Acotan que la miel, así llamada orgánica, pueda dar hasta $50 \mathrm{~kg}$ por lo que consideran que sí es rentable. Es por eso que según cuentan, a pesar que no se ha mantenido con tanta fuerza, la apicultura como actividad, continúa siendo redituable.

En este aspecto, Yucatán es el principal productor de miel del país, siendo los municipios del sur y oriente de Yucatán, los que sobresalen en este rubro (Tabla 1). Cabe mencionar que más del $90 \%$ de la producción es exportada al extranjero, principalmente a Europa, lo que indica la alta calidad que tiene la miel (GÜEMES et al., 2003).

TABLA 1: Producción de miel por toneladas por año, por municipio y por región del Estado de Yucatán, México.

\begin{tabular}{clrr}
\hline \multicolumn{1}{c}{ Región } & \multicolumn{1}{c}{ Municipio } & \multicolumn{1}{c}{ 2005* $^{*}$} & \multicolumn{1}{c}{ 2009** } \\
\hline \multirow{4}{*}{ Costa Norte } & Cansahcab & 47.05 & 0 \\
& Dzidzantún & 32.44 & 0 \\
& Dzilam de Bravo & 5.10 & 0 \\
& Dzilam González & 8.60 & 0 \\
& Sinanché & 1.01 & 7.45 \\
& Yobaín & 5.01 & 0 \\
\hline \multirow{4}{*}{ Sur } & Oxkutzcab & 81.21 & 326.97 \\
& Tekax & 330.4 & $1,004.53$ \\
& Tzucacab & 227.04 & 154.14 \\
\hline \multirow{4}{*}{ Oriente } & Calotmul & 99.50 & 151.95 \\
& Chemax & 281.7 & 195.46 \\
& Temozón & 92.50 & 177.7 \\
& Tizimín & 1087.00 & 522.43 \\
& Valladolid & 236 & 182.97 \\
\hline
\end{tabular}

* Fuente OIEDRUS (2006); ** Fuente INEGI 2009

\section{Entorno y depredadores}

Parte fundamental en el proceso de lograr mantener un apiario consiste en poder anticipar el ataque de animales depredadores, que puedan poner en riesgo la integridad de las abejas. Hormigas, iguanas, alacranes, 
serpientes, osos hormigueros, topos y cierto tipo de arácnidas son algunos de los depredadores más comunes.

Sin embargo, existen cierto tipo de animales, que son los que suelen atacar con mayor agudeza y efectividad las colmenas. Uno de los más agresivos es la hormiga. Se trata de insectos que suelen atacar a las abejas y que incluso puede llegar a exterminar un apiario completo. Por ello, una de las técnicas más empleadas por parte de los apicultores consiste en poner láminas debajo de las colmenas con la intención que por la anatomía del mismo insecto y la aleación metálica, éste resbale y le resulte imposible poder escalar hasta llegar a la colmena. Así, otro modo de mantener distanciadas a las hormigas, consiste en poner pequeños depósitos de agua, a especie de charcos, alrededor de donde se ubican las colmenas. En ese sentido, se tratan de poner dificultades y trampas que garanticen que algunos insectos depredadores se mantengan alejados y mueran antes de poder atacar a las abejas (PINKUS, 2013b).

"La hormiga no pasa en el agua, puede querer amontonarse, donde las crucetas son bajas, las hormigas son chingonas, hacen cerritos y cadenitas y ahí se van para arriba y el xulá (Echiton sp.) cuando es fuerte acaba casi un apiario, qué hace el xula, cuando ve apiario busca su cueva, hueco, tronco, albarrada en la noche ataca, de día se encueva. Vas a tu apiario, ya entró el $x$ ulá, ni modo, no viste donde se fue, en la noche vuelve a asomar. Puede diferenciar hueco de xulá, el xulá busca huecos grandes, hay diferencia entre iguanos o chihuó (Brachypelma spp.), el chihuó se mete en cualquier hueco, debajo de piedras, alacranes... los alacranes no nos afecta. Las arañas es malísimo par a nosotros, en la temporada que hay, por ejemplo en un árbol hacen su telaraña, cuando pase la abeja ahí se queda y baja la población de las colmenas. Ahora es época de arañas amarillas, perjudica, puedes ver que hay mucha floración, pero ahí se quedan las abejas, prendidas...30 mecates, como 600 metros de fondo. Hay otro tipo de hormigas que se llaman say (Atta cephalotes). Esas habitan en postes y cosas así, a veces en los pozos, dicen que el say es una hormiga que come de noche, en la noche sale a buscar hierbitas, hojas, dicen que cuando sale de día, porque a veces sale a buscar su comida, es porque se aproxima mal tiempo y por eso están adelantando a tener su comida, es señal de que algo del tiempo. Puede ser mal tiempo de cualquier tipo, no sólo lluvia. Por ejemplo en estos tiempos que abunda más el $\boldsymbol{x}$ ulá, lo que es octubre diciembre, porque son nortes, entonces cuando ven mucha hormiga es mal tiempo, va a cambiar mal tiempo, va a llover. Aquí se impide a que llegue, el agua impide que llegue la hormiga, porque no pasa en el agua. Pero el xulá es un depredador organizado, se detecta porque suena como un seseo a la distancia, se oye como viene bajo monte, se oye su ruido porque vienen como soldados, enfiladitos llegan, son guerreros".

Además de los peligros mencionados en los párrafos anteriores, también existen amenazas naturales para el buen funcionamiento y preservación de abejas y apiarios. Lluvias intensas, sequías, pero sobre todo huracanes, son factores que deben tomarse en cuenta para prevenir cualquier tipo de desastre. Por ello, en cuanto se tienen avisos de la cercanía de un fenómeno meteorológico que pueda afectar, se amarran con fuerza las cajas, las colmenas, de tal modo que aunque pudiesen caer por la fuerza de los vientos y el agua, no se rompan y contengan a las abejas adentro. A ello también obedece el que desde un inicio, los apiarios se coloquen en los "altillos", para que los flujos de agua no lleguen hasta donde se encuentran las abejas, pues en ocasiones, las crecientes de aguas pueden alcanzar metro y medio de altura.

Si el panal se moja, el moho que se produzca en él, provocará también que se reproduzcan bacterias dentro del mismo; y aunque la abeja esté sana y logre poner huevos, también se infectarán. Como es de suponer, tal situación impide el proceso de la producción y extracción de la miel, con lo que podría llegar a pérdidas que terminen con todo el ciclo de producción y por ende, con el apiario. En ese sentido, la preservación del entorno será fundamental para la apicultura, pues siempre que se cuente con flora variada y preservada, será incluso protección contra tempestades. Los apicultores nos cuentan que "los árboles son protección de los vientos, nortes, ciclones... los vientos normales, ya sea ciclón o algo, para que no me tiren las colmenas... la temperatura que les afecta es de calor a frío o de frío a calor, se mueren".

\section{Enfermedades culturales de las abejas}

En este sentido, algunos apicultores mencionan que las abejas pueden enfermarse de "fiebre" o de "diarrea", ello lo saben por el comportamiento que desarrollan los organismos. Para el primer caso, los apicultores 
mencionan que cuando las abejas no salen de las piqueras o están en el suelo tiradas dando vueltas es porque tienen fiebre, por lo que es necesario "airearlas", es decir, abrirles la tapa del alza superior, además de ponerles un tazón con agua y limón. El segundo diagnóstico lo dan cuando las abejas actúan erráticas "como borrachitas" y se ve que defecan muchas veces", al igual que en el primer caso, la cura es depositar dentro de la alza un tazón de agua y limón, así como azúcar. Es así que se da otra forma de antropomorfización, donde se relacionan padecimientos propios de los humanos con conductas - que los apicultores asumen -, son semejantes a los vistos en las abejas.

Si de peligros hablamos, quizá no sea del todo equivocado mencionar el daño que la gente cree, se puede adquirir cuando alguien echa alguna "maldición" sobre las tierras que trabaja, sin importar la actividad económica que se realice, pues aplica igual para ganaderos, que para horticultores, milperos o apicultores. El "hechizo" del terreno es una creencia arraigada entre los yucatecos, prácticamente en todas las regiones del estado. Se trata de la creencia que es posible que bajo el influjo del control de fuerzas sobre naturales, y en pacto con deidades asociadas al monte, el especialista ritual o $h$-men, tiene la capacidad de maldecir a base de conjuros un terreno determinado. Con ello, imposibilita que puedan ser productivas, que todo cuanto se siembre se muera o no de frutos, que incluso, en temporadas de lluvias, pueda llover en todos lados, con el vecino de al lado, pero no en el terreno de la persona sobre la cual se hizo la "maldad", este tema se ha abordado con mayor amplitud en el artículo "El monte y la cacería: construyendo espacios, transformando prácticas" (RODRÍGUEZ-BALAM, 2010a). Por eso, es necesario también saber a quién acudir para hacer la contra, para limpiar el terreno. Sobre esto señalan algunos: "Hay que llamar al shaman [Sic] para que venga. Aquí casi no hay h-menes, de otro lado los traen, los del sur lo traen porque sí tienen tradición de la primicia, sí. De hecho mi papá, casi no digamos que lo habla bien, pero lo entiende más no lo habla así".

El trabajo de los apicultores, así como la manera de entender el proceso de producción y extracción de la miel, inevitablemente conlleva generar ideas que tengan por finalidad comprender la etología de las abejas. Por ello, no es de extrañar que los apicultores le "hablen", que se refieran a ellas como "niñas" o "chamacas", que las saluden cada día y que les atribuyan incluso conductas o rasgos de personalidad, a partir de la interacción con los humanos. De hecho, si bien en maya existe una distinción lexical respecto a los animales domésticos, los de monte y los insectos, al respecto ver RODRÍGUEZ-BALAM (2010b), en este caso los apicultores afirman que la A. mellifera, al igual que cualquier otro animal, sienten y entienden la comunicación que se establece con ellas.

\begin{abstract}
"Hablo con las abejas, hola chamacas, como está, hola niñas... la abeja es como todo animal, conoce tu olor, tu voz, la vibración, la forma como lo tratas, es como cualquier animal, si lo golpeas tiene que reaccionar. Cuando llegas, lo primero que hace es observar, cómo está trabajando, ves que alguna no tiene entrada en la piquera, ah esa quien sabe qué tiene, vamos a ver qué es lo que le pasa, si todas están parejas ya, lo otro que cuando te acerques con el ahumador poquito de humo, con eso saben que hay alguien, que los trata bien, no te van a agredir, pero si llegas y destapas la tapa de golpe se van encima, si con poco de humo lo destapas no pasa nada. Les hablo, platico, como cualquier otro animal, nada de gritos. A veces cuando llego están secas las pilas, me da pena, me siento a darles agua. Calculo, que alguien llene las pilas porque es lo básico e indispensable, el agua. Tarda una semana, cuando venga nomás un poco les queda, cuando es tiempo de lluvia tarda más".
\end{abstract}

De acuerdo con lo narrado por apicultores, la manera de entender espacios, micro entornos y la etología animal en conjunto con la proyección de la dimensión humana, no se restringe al ámbito de los animales domésticos, como podría sugerir cierto tipo de prácticas culturales y conductas, bajo los esquemas de las sociedades contemporáneas. La capacidad intelectual de las abejas para organizarse, trabajar, socializar y las formas de generar interacción con los humanos es algo que los apicultores no se cuestionan. Es una idea que en cierto modo, se ha mantenido más o menos generalizada dentro de la construcción del entorno por parte de los sujetos que trabajan en el monte. Todo ello, cargado de significados culturales que se transmiten dentro de los procesos de socialización que se construyen en torno al ambiente. 
En la actualidad, los ritmos de la economía mundial ha contribuido a modificar de manera más acelerada las transformaciones del uso del suelo (si bien éstas se han dado a través de la historia), a la vez que han traído como consecuencia un cambio en la manera de relacionarse con el entorno, particularmente en la variedad de actividades que se contemplan como actividades a partir de las cuales obtener ingresos.

En el sur del estado de Yucatán, pero también en los poblados cercanos al litoral noreste, la milpa como cultivo homogéneo y la ganadería menor han tenido gran influencia en los impactos ambientales, como el Plan Chac (EASTMOND, 1991) y otras formas de extensionismo ganadero (RAMÍREZ; RIVERA, 2010). Con ello, llega también la inminente pérdida de conocimientos respecto al entorno, los nombres en lengua maya de las plantas y árboles, así como saberes históricos que han tenido para reconocer, interpretar y leer el paisaje que los rodea. No es extraño que los apicultores se quejen de los ganaderos, a quienes sólo les importa tumbar y cortar árboles para construir sus potreros, bardas y límites con la madera: "No saben qué es forraje, que florece y produce miel y néctar, sólo eso. Este es uno de los de sac catzín (Mimosa bahamensis) Benth. Ese sí florece pero no da néctar. No saben que hay poco cedro. El Chacah (Bursera simaruba) (L.) Sarg, es bueno, es melífera, pero no lo saben. Los ganaderos no saben ni qué es un piquete de abeja".

Por lo anterior, aunque son los menos, todavía existen algunos apicultores que se ocupan de transmitir este tipo de conocimientos y labores a sus hijos. Frente a la diversidad de mecanismos para la obtención de empleo, la migración a Mérida o la Riviera Maya para trabajar o estudiar, no es un asunto menor. En ese sentido, la pérdida de actividades vinculadas al monte como la apicultura y la agricultura, van cediendo terreno cada día. Por eso es que uno de los apicultores, quien también trabaja como maestro, después de su jornada en la escuela toma un descanso para esperar "que baje" el sol, e ir a ver a sus "niñas", las abejas. En el campo aprende de la vida, del entorno, y le da herramientas para introducir a su hijo los conocimientos del campo y la apicultura. Sobre ello nos relata:
“A mí me gusta mucho el campo, la vida, la disfruto más que en el pueblo. Por ejemplo, estoy en la escuela de 7 a 2 de la tarde y me fastidio, llego a mi casa, almuerzo y me acuesto un rato pero no estoy tranquilo, ya sea que me vaya aun apiario estoy más tranquilo, porque el sudar, cuando termines y llegas a tu casa te sientes tranquilo... en el campo he visto pájaros, águilas, y tábanos, que me recuerdan la canción de "estábamos matando tábanos" que era de Chico Ché.

Tengo un hijo de aproximadamente diez años, ya va a empezar a venir. Sí le voy a enseñar, si yo. ¿Cuál es la cosa de traerlo? Que cuando venga, tenga con qué trabajar, explicarle así como a ustedes, va a llegar un momento que diga que se quiere ir, al día siguiente volvemos a ir.

Mi hijo me ayuda en casa en la limpieza de los panales en... ya estoy pensando a partir de los doce años tráelo para que vaya aprendiendo, porque ya está más maduro para las picadas... ya lo picaron, en mi casa lo picaron una vez en el párpado, no le salió roncha nada, no es alérgico, eso tiene que ver si eres alérgico, porque el veneno va directo al corazón... hay picadas que te tira la fuerza, te bota pero unas que te pica y ni en cuenta, cuando hay flujo de néctar, mucha floración duele más, porque la abeja tiene más potencia, cuando no, el veneno es mínimo, no duele tanto, se distinga cuando tiene néctar, porque se infla, está llenita, cuando no, queda pequeña, eso se observa...

Es importante también que aprenda, que se acostumbre a los piquetes, eso es para ver si aguanta".

La esperanza es que los hijos puedan aprender los conocimientos que les transmiten los padres, que a su vez éstos hagan lo propio con sus hijos, para preservar así la extensión de prácticas y saberes a las generaciones futuras. Según advierten, no se trata sólo de "tradiciones", sino de una forma de reconocer el entorno, respetarlo y poder sacar de él los frutos que les otorga. Porque es "natural", porque "así lo han hecho los ancianos y los padres". En la educación de los hijos, cuenta un apicultor, es importante incluso enseñarles las brechas y caminos que años atrás hicieron los antiguos, porque "tienen historia". Hoy en día, nos dicen, cuando la flora y fauna ha sido modificada, es difícil encontrar la variedad de animales que antes podían ver. Por eso cuentan historias a sus hijos y nietos, de los tiempos pasados, cuando no era raro poder ver "cocodrilos junto con cultivos de maíz, calabaza y frijol”. 


\section{Consideraciones finales}

Sin duda alguna, la apicultura es quizás hoy en día una de las actividades productivas que más han acaparado la atención de especialistas. Biólogos, agrónomos así como economistas y grupos ecologistas, se han encargado de llamar la atención sobre los posibles daños o efectos que la desaparición de las abejas traerá como consecuencia del desequilibro ecológico que su extinción conlleva. Los discursos ecologistas, así como la gran demanda que ha tenido dentro del mercado la producción "artesanal", pero también la generación de mercados cada vez más demandantes respecto al origen, producción, difusión y venta de productos "amigables" con el ambiente que al mismo tiempo, dichas actividades podrían enmarcarse dentro de los esquemas de las industrias culturales. En ese sentido, la revalorización de la apicultura bajo tales referentes socioculturales, ha modificado en buena medida la manera como los apicultores yucatecos miran dicha actividad, pero también la importancia que le dedican a la transmisión del conocimiento a las nuevas generaciones.

Como lo hemos mencionado desde el inicio, nuestra propuesta en este artículo es la de presentar un acercamiento a través de la etnografía: observación participante, descripción de lo observado y entrevistas semiestruturadas. A partir de ello hemos podido recabar información que dé cuenta de la manera como la apicultura se mantiene a través del tiempo, con independencia de discursos "tradicionalistas" o "ecologistas". Su permanencia, con modificaciones y adaptaciones, se mantiene debido a que no sólo se le tiene por actividad económica, sino también, como elemento que porta aspectos vinculados a la historia, cultura, identidad, territorio y la relación con el entorno así como el interés por la preservación del mismo, consideración que ha preservado a los apicultores yucatecos, desde épocas remotas. La trascendencia e importancia histórica de la apicultura cuestiona la viabilidad de seguir considerando desde estudios antropológicos apegados a la preeminencia de la cultura, si la milpa y los rituales agrícolas son en realidad el elemento simbólico-cultural que da forma a la identidad y cosmovisiones de los grupos mayas peninsulares. Otras actividades, como la cacería o la apicultura, parecen no sólo tener más elementos con capacidad de cuestionar los ámbitos tradicionales descritos en las etnografías sobre mayas como generadores de cultura, sino también como prácticas que ponen en riesgo el ecosistema y con ello, la proyección cultural e identitaria de los hablantes de maya en la península yucateca.

\section{Agradecimientos}

Queremos agradecer a todos los informantes que nos ayudaron en este artículo. Así también al Dr. Eraldo Medeiros Costa Neto de la Universidade Estadual de Feira de Santana, Departamento de Ciências Biológicas por haber revisado el documento.

\section{Referencias}

ACEÑA, J. El sistema de comunicación de las abejas. Didáctica. Lengua y Literatura, Madrid, n. 2, p. 19-26, 1990.

AYALA, R. Revisión de las abejas sin aguijón de México (Hymenoptera: Apoidea). Apicultura Mexicana, Ciudad de México, v. 106, p 1-123,1999.

BARFIELD, T. Diccionario de Antropología. Ciudad de México: Ed Siglo XXI, 2000. 652 p.

BAUTISTA, F.; BATLLORI-SAMPEDRO, E.; PALACIO, G.; ORTÍZ-PÉREZ, M.; CASTILLO-GONZÁLEZ, M. Integración del conocimiento actual sobre los paisajes geomorfológicos de la Península de Yucatán. En: BAUTISTA F.; PALACIO G. (Ed.). Caracterización y manejo de los suelos de la Península de Yucatán: implicaciones agropecuarias, forestales y ambientales. Mérida: Universidad Autónoma de Campeche, Universidad Autónoma de Yucatán, 2005. p. 33- 58.

BERNARD, M. A. I.; LOZANO, M. C. Las bebidas sagradas Mayas: el balché y el saká. Sincronía, Guadalajara, v. 8, n. 28, 2003.

EASTMOND, A. Modernización agrícola y movilidad social hacia arriba en el sur de Yucatán. Nueva Antropología, Ciudad de México, v. 11, n. 39, p. 189-200, 1991.

ECHAZARRETA, C. Apicultura y producción de miel. En: DURÁN, R.; MÉNDEZ, M. (Ed.). Biodiversidad y desarrollo humano en Yucatán. Mérida: CICY, PPD-FMAM, CONABIO, SEDUMA, 2010. p. 109-11.

GÜEMES, F.; ECHAZARRETA, C.; VILLANUEVA, R.; PAT, J.; GÓMEZ, R. La apicultura en la península de Yucatán. Actividad de subsistencia en un entorno globalizado. Revista Mexicana del Caribe, Ciudad de México, v. 8, n. 16, p. 117-132, 2003.

INEGI. Anuario estadístico del estado de Yucatán. México, 2009. Versión electrónica.

LABOUGLE, R. J.; ZOZAYA, J. A. La apicultura en México, D.F. Ciencia y Desarrollo, Ciudad de México, v. 12, n. 69, p. 17-36, 1986. 
MANDUJANO, S.; RICO-GRAY, V. Hunting, use, and knowledge of the white-tailed deer (Odocoileus virginianus Hays) by the maya of central Yucatan, Mexico. Journal of Ethnobiology, Denton, v. 11, n. 2, p. 175-183, 1991.

MORALES, D. M. A. Hombre y medio ambiente en el pensamiento prehispánico. En: FOURNIER, P.; LÓPEZ, F. (Coord.). Patrimonio, identidad y complejidad social. Enfoques interdisciplinarios. Ciudad de México: Instituto Nacional de Antropología e Historia, 2010. p. 71-80.

OIEDRUS. Estadística básica. 2006. Disponible en: <http://www. campoyucatan.gob.mx/>. Fecha de consulta: 6 diciembre 2013.

PINKUS, R. M. Una mirada local de los artrópodos en Yucatán, México. Etnobiología, Ciudad de México, v. 11, n. 2, p. 58-68, 2013a.

PINKUS, R. M. Abejas, avispas y hormigas en la perspectiva local yucateca. Revista de Entomología Mexicana, Ciudad de México, v. 12, n. 2, p. 1706-1711, 2013 b.

QUEZADA, E. N. Biología y uso de las abejas sin aguijón de la Península de Yucatán, México. Hymenoptera. Meliponini. Ciudad de México: Universidad Autónoma de Yucatán, 2005. 112 p.

RAMÍREZ, L.; RIVERA, J. La ganadería en el contexto de la biodiversidad. En: DURÁN GARCÍA, R.; MÉNDEZ GONZÁLEZ, M. (Ed.). Biodiversidad y desarrollo humano en Yucatán. Mérida: CICY, PPD-FMAM, CONABIO, SEDUMA., 2010. p. 106-108.

RAMOS-ELORDUY, B. J.; COSTA-NETO, E. M.; LANDEROTORRES, I. Comparación de especies de abejas comestibles en la Sierra de Jibóia, (Bahia, Brasil) y Sierra de Zongolica (Veracruz, México). Revista Colombiana de Entomología, Bogotá, v. 35, n. 2, p. 217-223, 2009.

RIVERA, Z. A.; CAPPAS E SOUSA, J. P. Las abejas y la miel en los códices mayas (Códice Madrid o tro-cortertesiano). 1998 Disponible en: <http://www.culturaapicola.com.ar/apuntes/ historia/abejas20y20miel20en20los20mayas.PDF>.
RODRÍGUEZ-BALAM, E. El monte y la cacería: construyendo espacios, transformando prácticas. Revista Península, Mérida, v. 5, n. 2, p. 101-120, 2010a.

RODRÍGUEZ-BALAM, E. Pan Agrio maná del cielo: etnografía de los pentecostales en una comunidad de Yucatán. Mérida: CEPHCIS UNAM, 2010b. $182 \mathrm{p}$.

SANTOS-FITA, D.; NARANJO, E.; RANGEL-SALAZAR, J. Wildlife uses and hunting patterns in rural communities of the Yucatan Peninsula, Mexico. Journal of Ethnobiology and Ethnomedicine, London, v. 8, n. 38, 2012.

TERÁN, S.; RASMUSSEN, C. La milpa de los Mayas. Mérida: Ministerio de Relaciones Exteriores de Dinamarca (DANIDA), 1994. s/paginación.

TOLEDO, V.; BARRERA-BASSOLS, N.; GARCÍA-FRAPOLI, E.; ALARCÓN-CHAIRES, P. Uso múltiple y biodiversidad entre los mayas yucatecos (México). Interciencia, Caracas, v. 33, n. 5, p. 345-352, 2008.

TOLEDO, V.; BARRERA-BASSOLS, N.; GARCÍA-FRAPOLI, E.; ALARCÓN-CHAIRES, P. Etnoecología de los Mayas Yucatecos. Ciudad de México: Red de Etnoecología y Patrimonio Biocultural CONACYT, 2013. 26 p.

VANDAME, R.; COLIN, M.; OTERO, G. Abejas europeas y abejas africanizadas en México tolerancia a Varroa jacobsoni. [en línea]: Tercera parte: explicación de la tolerancia. 2000. Disponible en: <http://www.beekeeping.com/articulos/vandame/ vandame3_sp.htm>. Fecha de consulta: 5 diciembre 2013.

VILLANUEVA, R.; ROUBICK, D.; COLLI-UCÁN, W. Extinction of Melipona beecheii and traditional beekeeping in the Yucatan peninsula. Bee World, London, v. 86, n. 2, p. 35-41, 2005. 